PROCEEDINGS OF THE

AMERICAN MATHEMATICAL SOCIETY

Volume 128, Number 1, Pages 17-25

S 0002-9939(99)05292-2

Article electronically published on June 30, 1999

\title{
THE ROGERS-RAMANUJAN IDENTITIES, THE FINITE GENERAL LINEAR GROUPS, AND THE HALL-LITTLEWOOD POLYNOMIALS
}

\author{
JASON FULMAN
}

(Communicated by Ronald M. Solomon)

\begin{abstract}
We connect Gordon's generalization of the Rogers-Ramanujan identities with the Hall-Littlewood polynomials and with generating functions which arise in a probabilistic setting in the finite general linear groups. This yields a Rogers-Ramanujan type product formula for the $n \rightarrow \infty$ probability that an element of $G L(n, q)$ or $\operatorname{Mat}(n, q)$ is semisimple.
\end{abstract}

\section{BACKGROUND AND NOTATION}

The Rogers-Ramanujan identities are among the most remarkable partition identities in number theory and combinatorics. This paper will be concerned with the following generalization of the Rogers-Ramanujan identities, due to Gordon. Let $(x)_{n}$ denote $(1-x)\left(1-x^{2}\right) \cdots\left(1-x^{n}\right)$.

Theorem 1 ([A, page 111]). For $1 \leq i \leq k, k \geq 2$, and complex $x$ with $|x|<1$,

$$
\sum_{n_{1}, \cdots, n_{k-1} \geq 0} \frac{x^{N_{1}^{2}+\cdots+N_{k-1}^{2}+N_{i}+\cdots+N_{k-1}}}{(x)_{n_{1}} \cdots(x)_{n_{k-1}}}=\prod_{\substack{r=1 \\ r \neq 0, \pm i(\bmod 2 k+1)}}^{\infty} \frac{1}{1-x^{r}}
$$

where $N_{j}=n_{j}+\cdots+n_{k-1}$.

Gordon's generalization of the Rogers-Ramanujan identities has been widely studied and appears in many places in mathematics and physics. Andrews [A] discusses combinatorial aspects of these identities. In an important series of papers, Lepowsky and Wilson [LW1], [LW2], [LW3] connect the Gordon identities with affine Lie algebras and structures that they called $Z$-algebras (later interpreted as parafermion algebras in conformal field theory). Meurman and Primc [MP] solve a problem left open in [LW3], proving the independence of a $Z$-algebra basis and obtaining a $Z$-algebra proof of the Gordon identities. Feigin and Frenkel $[\mathrm{FF}]$ interpret the Gordon identities as a character formula for the Virasoro algebra. Andrews, Baxter, and Forrester $[\mathrm{ABF}]$ and Warnaar $[\mathrm{W}]$ relate the Gordon identities with statistical mechanics. For some number theoretic connections see the conference proceedings [AABRR].

We use the following standard notation from the theory of partitions. Call $\lambda=\left(\lambda_{1}, \lambda_{2}, \cdots\right)$ a partition of $n=|\lambda|$ if $\lambda_{1} \geq \lambda_{2} \geq \cdots \geq 0$ where the $\lambda_{i}$ are

Received by the editors March 6, 1998 .

1991 Mathematics Subject Classification. Primary 20P05, 05E05.

(C)1999 American Mathematical Society 
a sequence of positive integers stabilizing to 0 such that $\sum_{i} \lambda_{i}=n$. The $\lambda_{i}$ are referred to as the parts of $\lambda$. Let $m_{i}(\lambda)$ be the number of parts of $\lambda$ of size $i$, and set $\lambda_{i}^{\prime}=m_{i}(\lambda)+m_{i+1}(\lambda)+\cdots$. Define $n(\lambda)$ by $\sum_{i \geq 1}(i-1) \lambda_{i}$. Let $\left[u^{n}\right] f(u)$ denote the coefficient of $u^{n}$ in a power series $f(u)$.

\section{MAin RESUlts}

To begin we recall the Hall-Littlewood polynomials associated to a partition $\lambda$ (page 208 of [Mac]). Let $n$ be any integer such that $n \geq \lambda_{1}$. The permutation $w \in S_{n}$ acts on the variables $x_{1}, \cdots, x_{n}$ by sending $x_{i}$ to $x_{w(i)}$. Letting $t$ be a complex number, the Hall-Littlewood polynomials are defined as

$$
P_{\lambda}\left(x_{1}, \cdots, x_{n} ; t\right)=\frac{1}{\prod_{i \geq 0} \prod_{r=1}^{m_{i}(\lambda)} \frac{1-t^{r}}{1-t}} \sum_{w \in S_{n}} w\left(x_{1}^{\lambda_{1}} \cdots x_{n}^{\lambda_{n}} \prod_{i<j} \frac{x_{i}-t x_{j}}{x_{i}-x_{j}}\right) .
$$

At first glance it is not obvious that these are polynomials, but the denominators cancel out after the symmetrization. The Hall-Littlewood polynomials interpolate between the Schur functions $(t=0)$ and the monomial symmetric functions $(t=1)$.

Theorem 2. For $q>1$ and an integer $k \geq 2$,

$$
\begin{aligned}
& \sum_{\lambda: \lambda_{1}<k} \frac{P_{\lambda}\left(\frac{1}{q}, \frac{1}{q^{2}}, \frac{1}{q^{3}}, \frac{1}{q^{4}}, \cdots ; \frac{1}{q}\right)}{q^{n(\lambda)}}=\prod_{\substack{r=1 \\
r \neq 0, \pm k(\bmod 2 k+1)}}^{\infty}\left(\frac{1}{1-\frac{1}{q^{r}}}\right), \\
& \sum_{\lambda: \lambda_{1}<k} \frac{P_{\lambda}\left(\frac{1}{q^{2}}, \frac{1}{q^{3}}, \frac{1}{q^{4}}, \frac{1}{q^{5}}, \cdots ; \frac{1}{q}\right)}{q^{n(\lambda)}}=\prod_{\substack{r=1 \\
r \neq 0, \pm 1(\bmod 2 k+1)}}^{\infty}\left(\frac{1}{1-\frac{1}{q^{r}}}\right) .
\end{aligned}
$$

Proof. Macdonald's principal specialization formula (page 337 of [Mac]) states that

$$
P_{\lambda}\left(\frac{1}{q}, \frac{1}{q^{2}}, \frac{1}{q^{3}}, \frac{1}{q^{4}}, \cdots ; \frac{1}{q}\right)=\frac{1}{q^{|\lambda|+n(\lambda)}} \prod_{i} \frac{1}{\left(\frac{1}{q}\right)_{m_{i}(\lambda)}} .
$$

Combining this with the elementary fact that $n(\lambda)=\sum_{i}\left(\begin{array}{c}\lambda_{i}^{\prime} \\ 2\end{array}\right)$ shows that

$$
\frac{P_{\lambda}\left(\frac{1}{q}, \frac{1}{q^{2}}, \frac{1}{q^{3}}, \frac{1}{q^{4}}, \cdots ; \frac{1}{q}\right)}{q^{n(\lambda)}}=\frac{1}{q^{\sum_{i}\left(\lambda_{i}^{\prime}\right)^{2}} \prod_{i}\left(\frac{1}{q}\right)_{m_{i}(\lambda)}} .
$$

The first equality of the theorem follows by applying Theorem 1 with $i=k, x=$ $\frac{1}{q}, n_{j}=\lambda_{j}$ and $N_{j}=\lambda_{j}^{\prime}$. For the second equality, observe that for $u$ complex,

$$
\begin{aligned}
\frac{P_{\lambda}\left(\frac{u}{q}, \frac{u}{q^{2}}, \frac{u}{q^{3}}, \frac{u}{q^{4}}, \cdots ; \frac{1}{q}\right)}{q^{n(\lambda)}} & =u^{|\lambda|} \frac{P_{\lambda}\left(\frac{1}{q}, \frac{1}{q^{2}}, \frac{1}{q^{3}}, \frac{1}{q^{4}}, \cdots ; \frac{1}{q}\right)}{q^{n(\lambda)}} \\
& =\frac{u^{|\lambda|}}{q^{\sum_{i}\left(\lambda_{i}^{\prime}\right)^{2} \prod_{i}\left(\frac{1}{q}\right)_{m_{i}(\lambda)}}} .
\end{aligned}
$$

Now set $u=\frac{1}{q}$ and apply Theorem 1 with $i=1, x=\frac{1}{q}, n_{j}=\lambda_{j}$ and $N_{j}=\lambda_{j}^{\prime}$.

Remark. Stembridge [Ste] used properties of the Hall-Littlewood polynomials as a tool in giving proofs of the Rogers-Ramanujan identities. The statement of Theorem 2 gives a direct connection. 
Recall that the conjugacy classes of $G L(n, q)$ are parameterized by rational canonical form (Chapter 6 of Herstein $[\mathrm{H}]$ ). This form corresponds to the following combinatorial data. To each monic non-constant irreducible polynomial $\phi$ over a field of $q$ elements, associate a partition $\lambda_{\phi}$ of some non-negative integer $\left|\lambda_{\phi}\right|$. Let $\operatorname{deg}(\phi)$ denote the degree of $\phi$. This data represents a conjugacy class of $G L(n, q)$ if and only if $\left|\lambda_{z}\right|=0$ and $\sum_{\phi}\left|\lambda_{\phi}\right| \operatorname{deg}(\phi)=n$.

Definition. For $\alpha \in G L(n, q)$ and $\phi$ a monic, irreducible polynomial over $F_{q}$, a field of $q$ elements, define $\lambda_{\phi}(\alpha)$ to be the partition corresponding to the polynomial $\phi$ in the rational canonical form of $\alpha$.

The following elementary lemmas will be of use in studying the partitions $\lambda_{\phi}$.

Lemma 1. If the Taylor series of $f(u)$ around 0 converges at $u=1$, then

$$
\lim _{n \rightarrow \infty}\left[u^{n}\right] \frac{f(u)}{1-u}=f(1) .
$$

Proof. Write the Taylor expansion $f(u)=\sum_{n=0}^{\infty} a_{n} u^{n}$. Then observe that $\left[u^{n}\right] \frac{f(u)}{1-u}$ $=\sum_{i=0}^{n} a_{i}$.

Lemma 2. For $t \geq 1, q$ a prime power, and $u$ a formal variable,

$$
\prod_{\phi \text { irred. }}\left(1-\frac{u^{\operatorname{deg}(\phi)}}{q^{t \cdot \operatorname{deg}(\phi)}}\right)=1-\frac{u}{q^{t-1}} .
$$

Proof. Assume that $t=1$, the general case following by replacing $u$ with $\frac{u}{q^{t-1}}$. Expanding $\frac{1}{1-\frac{u}{q^{\operatorname{deg}(\phi)}(\phi)}}$ as a geometric series, unique factorization in $F_{q}[x]$ implies that the coefficient of $u^{d}$ in the reciprocal of the left hand side is $\frac{1}{q^{d}}$ times the number of monic polynomials of degree $d$, hence 1 . Comparing with the reciprocal of the right hand side proves the lemma.

Lemma 3. For $t \geq 1, q$ a prime power, and $u$ a formal variable,

$$
1-u=\prod_{\substack{\phi \neq z \\ \phi \text { irred. }}} \prod_{r=1}^{\infty}\left(1-\frac{u^{\operatorname{deg}(\phi)}}{q^{r \cdot d e g(\phi)}}\right) .
$$

Proof. By Lemma 2,

$$
\prod_{r=1}^{\infty}\left(1-\frac{u}{q^{r-1}}\right)=\prod_{\phi \text { irred. } r=1} \prod_{\text {ret }}^{\infty}\left(1-\frac{u^{\operatorname{deg}(\phi)}}{q^{r \cdot d e g(\phi)}}\right) .
$$

The lemma follows by cancelling the terms corresponding to $\phi=z$.

Lemma 4. For $q$ a prime power and $u$ complex with $|u| \leq 1$,

$$
\sum_{\lambda} \frac{u^{|\lambda|}}{q^{\sum_{i}\left(\lambda_{i}^{\prime}\right)^{2}} \prod_{i}\left(\frac{1}{q}\right)_{m_{i}(\lambda)}}=\prod_{r=1}^{\infty}\left(\frac{1}{1-\frac{u}{q^{r}}}\right) .
$$

Proof. On page 225 of [Mac] it is proved that

$$
\prod_{r=1}^{\infty}\left(1-x_{i}\right) \sum_{\lambda} t^{n(\lambda)} P_{\lambda}\left(x_{1}, x_{2}, x_{3}, \cdots ; t\right)=1 .
$$

Applying Macdonald's principal specialization formula with $t=\frac{1}{q}, x_{i}=\frac{u}{q^{i}}$ as in Theorem 2 proves the result. 
Lemma 5 (Euler). For $|q|>1$ and $u$ complex with $|u| \leq 1$,

1. $\prod_{r=1}^{\infty}\left(1-\frac{u}{q^{r}}\right)=\sum_{n=0}^{\infty} \frac{(-u)^{n}}{\left(q^{n}-1\right) \cdots(q-1)}$,

2. $\prod_{r=1}^{\infty}\left(\frac{1}{1-\frac{u}{q^{r}}}\right)=\sum_{n=0}^{\infty} \frac{\left.u^{n} q^{(} \begin{array}{c}n \\ 2\end{array}\right)}{\left(q^{n}-1\right) \cdots(q-1)}$.

Furthermore, these Taylor series converge at $u=1$.

Theorem 3 relates the Gordon identities with probability in the finite general linear groups.

Theorem 3. Let $\phi$ be a monic, irreducible polynomial over $F_{q}$. Let $k \geq 2$ be an integer. Then the $n \rightarrow \infty$ limit of the chance that a uniformly chosen element $\alpha$ of $G L(n, q)$ has the largest part of the partition $\lambda_{\phi}(\alpha)$ less than $k$ is equal to

$$
\prod_{\substack{r=1 \\ r=0, \pm k(\bmod 2 k+1)}}^{\infty}\left(1-\frac{1}{q^{r \cdot d e g}(\phi)}\right) .
$$

Proof. Assume for simplicity that $\phi=z-1$. From the proof it will be clear that the general case follows. Stong [Sto] used Kung's [Ku] formula for the sizes of the conjugacy classes of $G L(n, q)$ to find a "cycle index" for the general linear groups. Using the notation

$d_{i}(\lambda)=1 m_{1}(\lambda)+2 m_{2}(\lambda)+\cdots+(i-1) m_{i-1}(\lambda)+i\left(m_{i}(\lambda)+m_{i+1}(\lambda)+\cdots+m_{j}(\lambda)\right)$,

he obtained the equality

$$
\begin{aligned}
1+\sum_{n=1}^{\infty} & \frac{u^{n}}{|G L(n, q)|} \sum_{\alpha \in G L(n, q)} \prod_{\substack{\phi \neq z \\
\text { irred. }}} x_{\phi, \lambda_{\phi}(\alpha)} \\
& =\prod_{\substack{\phi \neq z \\
\phi \text { irred. }}}\left[\sum_{\lambda} x_{\phi, \lambda} \frac{u^{|\lambda| \operatorname{deg}(\phi)}}{\prod_{i} \prod_{k=1}^{m_{i}(\lambda)}\left(q^{\operatorname{deg}(\phi) d_{i}}-q^{\operatorname{deg}(\phi)\left(d_{i}-k\right)}\right)}\right] .
\end{aligned}
$$

Observe that

$$
\begin{aligned}
\prod_{i} \prod_{k=1}^{m_{i}}\left(q^{d_{i}}-q^{\left(d_{i}-k\right)}\right) & =q^{\sum_{i} m_{i}(\lambda) d_{i}(\lambda)} \prod_{i}\left(\frac{1}{q}\right)_{m_{i}(\lambda)} \\
& =q^{\sum_{i} m_{i}(\lambda)\left[\left(\sum_{h<i} h m_{h}(\lambda)\right)+i m_{i}(\lambda)+\sum_{i<k} i m_{k}(\lambda)\right]} \prod_{i}\left(\frac{1}{q}\right)_{m_{i}(\lambda)} \\
& =q^{\sum_{i}\left[i m_{i}(\lambda)^{2}+2 m_{i}(\lambda) \sum_{h<i} h m_{h}(\lambda)\right]} \prod_{i}\left(\frac{1}{q}\right)_{m_{i}(\lambda)} \\
& =q^{\sum_{i}\left[\sum_{h<i} m_{h}(\lambda)\right]^{2}} \prod_{i}\left(\frac{1}{q}\right)_{m_{i}(\lambda)} \\
& =q^{\sum_{i}\left(\lambda_{i}^{\prime}\right)^{2}} \prod_{i}\left(\frac{1}{q}\right)_{m_{i}(\lambda)}
\end{aligned}
$$


Combining this observation with Lemma 3 shows that

$$
\begin{aligned}
& 1+\sum_{n=1}^{\infty} \frac{u^{n}}{|G L(n, q)|} \sum_{\alpha \in G L(n, q)} \prod_{\substack{\phi \neq z \\
\text { irred. }}} x_{\phi, \lambda_{\phi}(\alpha)} \\
= & \frac{1}{1-u} \prod_{\substack{\phi \neq z \\
\phi \text { irred. }}}\left[\prod_{r=1}^{\infty}\left(1-\frac{u^{\operatorname{deg}(\phi)}}{q^{r \cdot \operatorname{deg}(\phi)}}\right)\right]\left[\sum_{\lambda} x_{\phi, \lambda} \frac{u^{|\lambda| \operatorname{deg}(\phi)}}{q^{\operatorname{deg}(\phi) \sum_{i}\left(\lambda_{i}^{\prime}\right)^{2}} \prod_{i}\left(\frac{1}{q^{d e g(\phi)}}\right)_{m_{i}(\lambda)}}\right] .
\end{aligned}
$$

Setting $x_{z-1, \lambda}=0$ if the largest part of $\lambda$ is greater than or equal to $k$, and all $x_{\phi, \lambda}=1$ otherwise shows by Lemma 4 that the sought probability is

$$
\lim _{n \rightarrow \infty}\left[u^{n}\right] \frac{1}{1-u}\left(\prod_{r=1}^{\infty}\left(1-\frac{u}{q^{r}}\right)\right)\left(\sum_{\lambda: \lambda_{1}<k} \frac{u^{|\lambda|}}{q^{\sum_{i}\left(\lambda_{i}^{\prime}\right)^{2}} \prod_{i}\left(\frac{1}{q}\right)_{m_{i}(\lambda)}}\right) .
$$

By Lemmas 4 and 5, Lemma 1 applies and

$$
\begin{aligned}
& \lim _{n \rightarrow \infty}\left[u^{n}\right] \frac{1}{1-u}\left(\prod_{r=1}^{\infty}\left(1-\frac{u}{q^{r}}\right)\right)\left(\sum_{\lambda: \lambda_{1}<k} \frac{u^{|\lambda|}}{q^{\sum_{i}\left(\lambda_{i}^{\prime}\right)^{2}} \prod_{i}\left(\frac{1}{q}\right)_{m_{i}(\lambda)}}\right) \\
= & \left(\prod_{r=1}^{\infty}\left(1-\frac{1}{q^{r}}\right)\right)\left(\sum_{\lambda: \lambda_{1}<k} \frac{1}{q^{\sum_{i}\left(\lambda_{i}^{\prime}\right)^{2}} \prod_{i}\left(\frac{1}{q}\right)_{m_{i}(\lambda)}}\right) .
\end{aligned}
$$

The theorem follows from the Gordon identities with $i=k, x=\frac{1}{q}, n_{j}=m_{j}(\lambda)$, and $N_{j}=\lambda_{j}^{\prime}$.

Let $\operatorname{Mat}(n, q)$ denote the set of $n \times n$ matrices with entries in the finite field $F_{q}$. Recall that $\alpha \in \operatorname{Mat}(n, q)$ is said to be semisimple if it is diagonalizable over the algebraic closure $\bar{F}_{q}$.

Theorem 4. The $n \rightarrow \infty$ limiting probability that an element of $G L(n, q)$ is semisimple is

$$
\prod_{\substack{r=1 \\ r=0, \pm 2(\bmod 5)}}^{\infty} \frac{\left(1-\frac{1}{q^{r-1}}\right)}{\left(1-\frac{1}{q^{r}}\right)} .
$$

Proof. From the theory of Jordan canonical forms, an element $\alpha$ of $G L(n, q)$ is semisimple if and only if the largest part of $\lambda_{\phi}(\alpha)$ is less than two for all $\phi$. Thus by the cycle index for $G L(n, q)$ (see the proof of Theorem 3) and Lemma 3, the sought probability is

$$
\begin{aligned}
& \lim _{n \rightarrow \infty}\left[u^{n}\right] \prod_{\substack{\phi \neq z \\
\phi \text { irred. }}} \sum_{\lambda: \lambda_{1}<2} \frac{u^{\operatorname{deg}(\phi)|\lambda|}}{q^{\operatorname{deg}(\phi) \sum_{i}\left(\lambda_{i}^{\prime}\right)^{2}} \prod_{i}\left(\frac{1}{q^{d e g(\phi)}}\right)_{m_{i}(\lambda)}} \\
& =\lim _{n \rightarrow \infty}\left[u^{n}\right] \frac{1}{1-u} \prod_{\substack{\phi \neq z \\
\text { irred. }}}\left(\prod_{r=1}^{\infty}\left(1-\frac{u^{\operatorname{deg}(\phi)}}{q^{r \cdot \operatorname{deg}(\phi)}}\right)\right) \\
& \cdot\left(\sum_{\lambda: \lambda_{1}<2} \frac{u^{\operatorname{deg}(\phi)|\lambda|}}{q^{\operatorname{deg}(\phi) \sum_{i}\left(\lambda_{i}^{\prime}\right)^{2}} \prod_{i}\left(\frac{1}{q^{\operatorname{deg}(\phi)}}\right)_{m_{i}(\lambda)}}\right) .
\end{aligned}
$$


By Theorem 1 with $i=1, k=2, x=\frac{1}{q^{\operatorname{deg}(\phi)}}, n_{j}=\lambda_{j}, N_{j}=\lambda_{j}^{\prime}$ and Lemma 2 , the desired probability becomes

$$
\begin{aligned}
& \prod_{\substack{\phi \neq z \\
\phi \text { irred. }}} \prod_{\substack{r=1 \\
r=0, \pm 2(\bmod 5)}}^{\infty}\left(1-\frac{1}{q^{r \cdot \operatorname{deg}(\phi)}}\right) \\
& =\prod_{\substack{r=1 \\
r=0, \pm 2(\bmod 5)}}^{\infty}\left(\frac{1}{1-\frac{1}{q^{r}}}\right) \prod_{\phi \text { irred. }} \prod_{\substack{r=1 \\
r=0, \pm 2(\bmod 5)}}^{\infty}\left(1-\frac{1}{q^{r \cdot \operatorname{deg}(\phi)}}\right) \\
& =\prod_{\substack{r=1 \\
r=0, \pm 2(\bmod 5)}}^{\infty} \frac{\left(1-\frac{1}{q^{r-1}}\right)}{\left(1-\frac{1}{q^{r}}\right)}
\end{aligned}
$$

Theorem 5. The $n \rightarrow \infty$ limiting probability that an element of $\operatorname{Mat}(n, q)$ is semisimple is

$$
\prod_{\substack{r=1 \\ r=0, \pm 2(\bmod 5)}}^{\infty}\left(1-\frac{1}{q^{r-1}}\right)
$$

Proof. The orbits of $G L(n, q)$ on $\operatorname{Mat}(n, q)$ under conjugation are also parameterized by the data $\lambda_{\phi}$. However the polynomial $z$ may appear with non-zero multiplicity, so the restriction that $\left|\lambda_{z}\right|=0$ does not apply. Stong [Sto] obtained the generating function equality

$$
\begin{aligned}
& 1+\sum_{n=1}^{\infty} \frac{u^{n}}{|G L(n, q)|} \sum_{\alpha \in M a t(n, q)} \prod_{\phi \text { irred. }} x_{\phi, \lambda_{\phi}(\alpha)} \\
& \quad=\prod_{\phi \text { irred. }}\left[\sum_{\lambda} x_{\phi, \lambda} \frac{u^{|\lambda| \operatorname{deg}(\phi)}}{\prod_{i} \prod_{k=1}^{m_{i}(\lambda)}\left(q^{\operatorname{deg}(\phi) d_{i}}-q^{\operatorname{deg}(\phi)\left(d_{i}-k\right)}\right)}\right],
\end{aligned}
$$

where $d_{i}(\lambda)$ is defined by

$$
\begin{aligned}
d_{i}(\lambda)= & 1 m_{1}(\lambda)+2 m_{2}(\lambda)+\cdots+(i-1) m_{i-1}(\lambda) \\
& +i\left(m_{i}(\lambda)+m_{i+1}(\lambda)+\cdots+m_{j}(\lambda)\right) .
\end{aligned}
$$

Manipulations identical to those in Theorem 3 show that

$$
\begin{aligned}
& 1+\sum_{n=1}^{\infty} \frac{u^{n}}{|G L(n, q)|} \sum_{\alpha \in M a t(n, q)} \prod_{\text {irred. }} x_{\phi, \lambda_{\phi}(\alpha)} \\
= & \frac{1}{1-u} \prod_{\phi \text { irred. }}\left[\prod_{r=1}^{\infty}\left(1-\frac{u^{\operatorname{deg}(\phi)}}{q^{r \cdot \operatorname{deg}(\phi)}}\right)\right]\left[\sum_{\lambda} x_{\phi, \lambda} \frac{u^{|\lambda| \operatorname{deg}(\phi)}}{q^{\operatorname{deg}(\phi) \sum_{i}\left(\lambda_{i}^{\prime}\right)^{2}} \prod_{i}\left(\frac{1}{q^{\operatorname{deg}(\phi)}}\right)_{m_{i}(\lambda)}}\right] .
\end{aligned}
$$


Arguing as in Theorem 4 shows that the $n \rightarrow \infty$ limit of the chance that an element of $\operatorname{Mat}(n, q)$ is semisimple is

$$
\begin{aligned}
& \lim _{n \rightarrow \infty} \frac{|G L(n, q)|}{q^{n^{2}}}\left[u^{n}\right] \prod_{\phi} \sum_{\text {irred. } \lambda: \lambda_{1}<2} \frac{u^{\operatorname{deg}(\phi)|\lambda|}}{q^{\operatorname{deg}(\phi) \sum_{i}\left(\lambda_{i}^{\prime}\right)^{2}} \prod_{i}\left(\frac{1}{q^{\operatorname{deg}(\phi)}}\right)_{m_{i}(\lambda)}} \\
& =\lim _{n \rightarrow \infty} \frac{|G L(n, q)|}{q^{n^{2}}}\left[u^{n}\right] \frac{1}{1-u} \frac{1}{\prod_{r=1}^{\infty}\left(1-\frac{u}{q^{r}}\right)} \prod_{\phi \text { irred. }} \\
& {\left[\prod_{r=1}^{\infty}\left(1-\frac{u^{\operatorname{deg}(\phi)}}{q^{r \cdot \operatorname{deg}(\phi)}}\right) \sum_{\lambda: \lambda_{1}<2} \frac{u^{\operatorname{deg}(\phi)|\lambda|}}{q^{\operatorname{deg}(\phi) \sum_{i}\left(\lambda_{i}^{\prime}\right)^{2}} \prod_{i}\left(\frac{1}{q^{\operatorname{deg}(\phi)}}\right)_{m_{i}}(\lambda)}\right]} \\
& =\prod_{\phi \text { irred. }}\left[\prod_{r=1}^{\infty}\left(1-\frac{1}{q^{r \cdot d e g}(\phi)}\right)\right]\left[\sum_{\lambda: \lambda_{1}<2} \frac{1}{q^{\operatorname{deg}(\phi) \sum_{i}\left(\lambda_{i}^{\prime}\right)^{2}} \prod_{i}\left(\frac{1}{q^{\operatorname{deg}(\phi)}}\right)_{m_{i}(\lambda)}}\right] \\
& =\prod_{\phi \text { irred. }} \prod_{\substack{r=1 \\
r=0, \pm 2(\bmod 5)}}^{\infty}\left(1-\frac{1}{q^{r \cdot \operatorname{deg}(\phi)}}\right)=\prod_{\substack{r=1 \\
r=0, \pm 2(\bmod 5)}}^{\infty}\left(1-\frac{1}{q^{r-1}}\right) .
\end{aligned}
$$

Theorems 2 and 3 suggest a connection between the Hall-Littlewood polynomials and the finite general linear groups. Theorem 6 makes this connection precise.

Theorem 6. For q a prime power and u a formal variable,

$$
\begin{aligned}
& (1-u)\left[1+\sum_{n=1}^{\infty} \frac{u^{n}}{|G L(n, q)|} \sum_{\alpha \in G L(n, q)} \prod_{\substack{\phi \neq \neq z \\
\phi \text { irred. }}} x_{\phi, \lambda_{\phi}(\alpha)}\right] \\
= & \prod_{\substack{\phi \neq z \\
\phi \text { irred. }}} \prod_{r=1}^{\infty}\left(1-\frac{u^{\operatorname{deg}(\phi)}}{q^{r \cdot \operatorname{deg}(\phi)}}\right)\left[\frac{P_{\lambda}\left(\left(\frac{u}{q}\right)^{\operatorname{deg}(\phi)},\left(\frac{u}{q}\right)^{2 \operatorname{deg}(\phi)},\left(\frac{u}{q}\right)^{3 \operatorname{deg}(\phi)}, \cdots ;\left(\frac{1}{q}\right)^{\operatorname{deg}(\phi)}\right)}{q^{n(\lambda) \cdot \operatorname{deg}(\phi)}}\right] .
\end{aligned}
$$

Proof. The proof of Theorem 3 contained the equality

$$
\begin{aligned}
& (1-u)\left[1+\sum_{n=1}^{\infty} \frac{u^{n}}{|G L(n, q)|} \sum_{\alpha \in G L(n, q)} \prod_{\substack{\phi \neq z \\
\phi \text { irred. }}} x_{\phi, \lambda_{\phi}(\alpha)}\right] \\
= & \prod_{\substack{\phi \neq z \\
\phi \text { irred. }}}\left[\prod_{r=1}^{\infty}\left(1-\frac{u^{\operatorname{deg}(\phi)}}{q^{r \cdot \operatorname{deg}(\phi)}}\right)\right]\left[\sum_{\lambda} x_{\phi, \lambda} \frac{u^{|\lambda| \operatorname{deg}(\phi)}}{q^{\operatorname{deg}(\phi) \sum_{i}\left(\lambda_{i}^{\prime}\right)^{2}} \prod_{i}\left(\frac{1}{q^{\operatorname{deg}(\phi)}}\right)_{m_{i}(\lambda)}}\right] .
\end{aligned}
$$

The theorem now follows from the use of Macdonald's principal specialization formula (as in Theorem 2) to conclude that

$$
\frac{P_{\lambda}\left(\frac{u}{q}, \frac{u}{q^{2}}, \frac{u}{q^{3}}, \frac{u}{q^{4}}, \cdots ; \frac{1}{q}\right)}{q^{n(\lambda)}}=\frac{u^{|\lambda|}}{q^{\sum_{i}\left(\lambda_{i}^{\prime}\right)^{2}} \prod_{i}\left(\frac{1}{q}\right)_{m_{i}(\lambda)}} .
$$

Remark. Although Theorem 6 followed easily from techniques in Theorems 2 and 3 , its formulation admits an attractive probabilistic interpretation. Fix $u$ such that $0<u<1$ and fix an irreducible monic polynomial $\phi \neq z$. Then pick a natural number with probability of getting $n$ equal to $(1-u) u^{n}$. Finally, choose $\alpha$ uniformly at random in $G L(n, q)$ and let $\lambda_{\phi}(\alpha)$ be the random partition so defined. This procedure induces a probability measure on the set of all partitions of all integers. 
Theorem 6 implies that measures obtained for different $\phi$ are independent, and that the mass the measure for a given $\phi$ assigns to a partition $\lambda$ is equal to

$$
\prod_{r=1}^{\infty}\left(1-\frac{u^{\operatorname{deg}(\phi)}}{q^{r \cdot \operatorname{deg}(\phi)}}\right)\left[\frac{P_{\lambda}\left(\left(\frac{u}{q}\right)^{\operatorname{deg}(\phi)},\left(\frac{u}{q}\right)^{2 \operatorname{deg}(\phi)},\left(\frac{u}{q}\right)^{3 \operatorname{deg}(\phi)}, \cdots ;\left(\frac{1}{q}\right)^{\operatorname{deg}(\phi)}\right)}{q^{n(\lambda) \cdot \operatorname{deg}(\phi)}}\right] .
$$

This connection with symmetric functions leads to a probabilistic algorithm for growing the random partitions $\lambda_{\phi}$. This viewpoint is used in [Fu1] to give probabilistic proofs of some group theoretic results of Steinberg, Rudvalis/Shinoda, and Lusztig. Cycle indices for the finite unitary, symplectic, and orthogonal groups appear in [Fu2].

\section{Conclusion}

This paper has offered connections between Gordon's generalization of the Rogers-Ramanujan identities, the Hall-Littlewood polynomials, and generating functions which arise in the study of the finite general linear groups. The following questions seem natural.

- Are there analogs of the Gordon identities for the finite unitary, symplectic, and orthogonal groups? The Gordon identities do play a role in Misra's work on affine symplectic Lie algebras [Mi1] and in Mandia's work [Man] on the affine Lie algebras $B_{l}^{(1)}, F_{4}^{(1)}$, and $G_{2}^{(1)}$. Misra has also used $Z$-algebra methods to relate the Gordon identities to $\widetilde{A_{n}}$.

- Ian Macdonald has suggested that the results of this paper should carry over to the affine finite general linear groups. The Hall-Littlewood polynomials have affine analogs [EK].

- It is known (e.g. Kac [Ka]) that the product side of the Rogers-Ramanujan identities has interesting modular properties. Can this phenomenon be understood group theoretically in terms of the conjugacy classes of the general linear groups; i.e. is there "general linear group moonshine"? In this regard note that Jing [J1, J2] has connected the Hall-Littlewood polynomials with vertex operators.

\section{ACKNOWLEDGEMENTS}

This work is taken from the author's Ph.D. thesis, done under the supervision of Persi Diaconis. His idea of studying the random partitions $\lambda_{\phi}$ led to this work. We thank Ed Frenkel, Jim Lepowsky, and the referee for providing pointers to the Rogers-Ramanujan literature. This research was done under the generous 3-year support of the National Defense Science and Engineering Graduate Fellowship and the support of the Alfred P. Sloan Foundation Dissertation Fellowship.

\section{REFERENCES}

[A] Andrews, G., The theory of partitions. Encyclopedia of Mathematics and its Applications, Vol. 2. Addison-Wesley Publishing Co., Reading, Mass.-London-Amsterdam, 1976. MR 58:27738

[AABRR] Andrews, G., Askey, R., Berndt, B., Ramanathan, K., and Rankin, R., Ramanujan revisited. Proceedings of the Ramanujan Centenary Conference, Academic Press, Inc., Boston, 1988. MR 89c: 11001 
[ABF] Andrews, G., Baxter, R. J., and Forrester, P. J.: Eight-vertex SOS model and generalized Rogers-Ramanujan type identities, J. Stat. Phys. 35 (1984), 193-266. MR 86a: 82001

[EK] Etingof, P. and Kirillov, A., On the affine analogue of Jack and Macdonald polynomials, Duke Math. J. 78 (1995), 229-256. MR 97k:17035

[FF] Feigin, B. and Frenkel, E.: Coinvariants of nilpotent subalgebras of the Virasoro algebra and partition identities. I. M. Gelfand Seminar, Adv. Soviet Math. 16, Part 1, 139-148, Amer. Math. Soc., Providence, RI, 1993. MR 94g:17054

[Fu1] Fulman, J., A probabilistic approach toward conjugacy classes in the finite general linear and unitary groups, J. Algebra 212 (1999), 557-590. CMP 99:09

[Fu2] Fulman, J., Cycle indices for the finite classical groups, J. Group Theory 2 (1999), 251-289.

[H] Herstein, I.N., Topics in algebra. Second edition. Xerox College Publishing, Lexington, Mass. - Toronto, Ont., 1975. MR 50:9456

[J1] Jing, N., Vertex operators and Hall-Littlewood symmetric functions, Advances in Math. 87 (1991), 226-248. MR 93c:17039

[J2] Jing, N. Boson-fermion correspondence for Hall-Littlewood polynomials, J. Math. Physics 36 (1995), 7073-7080. MR 96m:17049

[Ka] Kac, V.G., Modular invariance in mathematics and physics. American Mathematical Society centennial publications, Vol. II (Providence, RI, 1988), 337-350, Amer. Math. Soc., Providence, RI, 1992. MR 93h:17061

[Ku] Kung, J., The cycle structure of a linear transformation over a finite field, Lin. Alg. Appl. 36 (1981), 141-155. MR 82d:15012

[LW1] Lepowsky, J. and Wilson, R. L., A new family of algebras underlying the RogersRamanujan identities and generalizations, Proc. Nat. Acad. Sci. USA 78 (1981), 72547258. MR 82k:10016

[LW2] Lepowsky, J. and Wilson, R.L., The structure of standard modules, I: Universal algebras and the Rogers-Ramanujan identities, Invent. Math. 77 (1984), 199-290. MR 85m: 17008

[LW3] Lepowsky, J. and Wilson, R.L., The structure of standard modules, II: The case $A_{1}^{(1)}$ principal gradation, Invent. Math. 79 (1985), 417-442. MR 86g:17014

[Mac] Macdonald, I.G., Symmetric functions and Hall polynomials. Second Edition. Clarendon Press, Oxford, 1995. MR 96h:05207

[Man] Mandia, M., Structure of the level one standard modules for the affine Lie algebras $B_{l}^{(1)}, F_{4}^{(1)}$, and $G_{2}^{(1)}$, Memoirs American Math. Soc. 362, 1987. MR 88h:17023

[Mi1] Misra, K., Level one standard modules for affine symplectic Lie algebras, Math. Ann. 287 (1990), 287-302. MR 91j:17036

[Mi2] Misra, K., Level two standard $\widetilde{A_{n}}$-modules, J. Algebra 137 (1991), 56-76. MR 92a: 17040

[MP] Meurman, A. and Primc, M., Annihilating ideals of standard modules of $\underline{s l}(2, C) \sim$ and combinatorial identities, Advances in Math. 64 (1987), 177-240. MR 89c:17031

[Ste] Stembridge, J.: Hall-Littlewood functions, plane partitions, and the Rogers-Ramanujan identities, Trans. Amer. Math. Soc. 319 (1990), 469-498. MR 90j:05021

[Sto] Stong, R., Some asymptotic results on finite vector spaces, Adv. Appl. Math. 9 (1988), 167-199. MR 89c:05007

[W] Warnaar, S. Ole, The Andrews-Gordon identities and q-multinomial coefficients, Commun. Math. Phys. 184 (1997), 203-232. MR 98m:11108

Department of Mathematics, Dartmouth College, Hanover, New Hampshire 037553551

E-mail address: Fulman@Dartmouth.Edu 